\title{
JStuff - a preliminary extragalactic model for the ESA-Gaia satellite simulation framework
}

\author{
A. G. O. Krone-Martins ${ }^{1,2}$, C. Ducourant ${ }^{1}$, \\ R. Teixeira ${ }^{2}$ and X. Luri ${ }^{3}$ \\ ${ }^{1}$ Observatoire de Sciences d'Aquitaine, Université de Bordeaux I \\ 2, rue de l'Observatoire, Floirac, France \\ email: krone@obs.u-bordeaux1.fr \\ ${ }^{2}$ Inst. de Astron. Geof. e Ciên. Atm., Universidade de São Paulo, \\ Rua do Matão, 1226, 05508-900, Cidade Universitária, São Paulo, Brazil \\ ${ }^{3}$ Dept. d'Astronomia i Meteorologia, Universitat de Barcelona, Martí Franquès S/N, Barcelona,
}

\begin{abstract}
In this work we describe the JStuff, a preliminary generator of mock catalogues of extragalactic objects based on the Stuff code. This version is being implemented in the Java language for the ESA-Gaia satellite simulation framework. We also compare some results obtained with both versions of the simulator.
\end{abstract}

Keywords. catalogs, galaxies: fundamental parameters, galaxies: luminosity function

\section{Introduction}

The ESA-Gaia mission, Perryman (2005), is one of the most ambitious projects of the modern astronomy. With the satellite launch date fixed at the end of 2011, the final all-sky catalog shall be released sometime before 2020. The satellite observations and the reduction procedures will result into an impressive terabyte class all-sky catalog, complete up to $\mathrm{G}=20$, with more than one billion objects of all types with amazing astrometric precision $(7 \mu$ as at $\mathrm{V}<13,25 \mu$ as at $\mathrm{V}<15$ and a fraction of mas at $\mathrm{V}<20,11$ million paralaxes with errors smaller than $1 \%$, and 150 million in the $10 \%$ range), spectrophotometric data spread over 66 pixels $(330-1000 \mathrm{~nm})$ and high-resolution spectrometric data in the CaII-triplet region $(847-874 \mathrm{~nm}, 0.026 \mathrm{~nm} / \mathrm{pix})$.

In order to design the reduction and analysis algorithms, it is necessary to implement a realistic mission simulation environment. The GaiaSimulator, Altamirano et al. (2005), is the official framework where all the simulation packages are built-in: it includes a pixel-level simulation package, a telemetry stream simulation package, a statistically simulated catalog generator, and the universe model simulators: Galaxy, Extragalactic, Solar System. JStuff is the first implementation in Java of the code to generate catalogs of unresolved galaxies.

\section{The simulation algorithm}

This version of JStuff is heavily based on the Stuff code first described in Bertin \& Arnouts (1996). It simulates a mock catalog of galaxies at a given region of the sky, at this time using a positional sampling that is uniformly random. Nonetheless it is planned as a possible future improvement, the implementation of a two-point correlation function.

In order to simulate the galaxies parameters, the code first sample randomly, from a poissonian distribution, the number of galaxies of Hubble type (E, S0, Sab, Sbc, Scd, and Irr are implemented), assuming Schechter (1976) luminosity functions. Then, each galaxy 

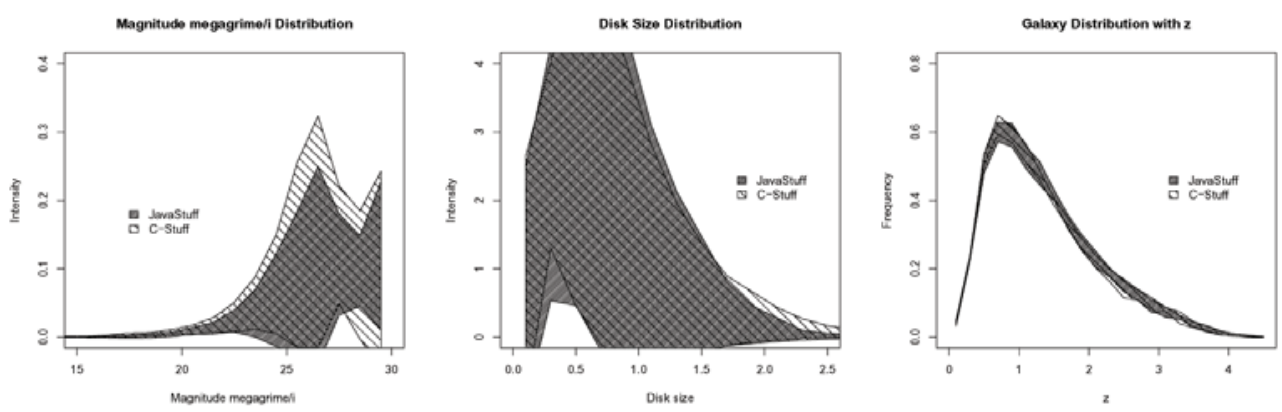

Figure 1. Comparison of the results obtained with the JStuff and the Stuff codes for three parameters. The confidence intervals are at the three sigma level.

is assembled as a sum of two components, a spheroidal (following a de Vaucouleurs law) and a disk-like one.

For elliptical galaxies, the adopted relation between the absolute B magnitude and effective radius is the one described in Binggelli et al. 1984. The spheroid flattening is randomly selected from a normal distribution, from Sandage et al. (1970). The disk component is simulated with an exponential profile. The central surface brightness is randomly selected from a gaussian distribution, and the disk inclination and position angle are also randomly selected.

The code simulates the internal disk extinction in the observed galaxy, using a curve obtained for the LMC. We apply Metcalfe et al. (1991) K+e corrections with polynomial fits. Finally, each galaxy is placed at a redshift (and its bolometric luminosity and angular diameter distance are calculated). Spectra is generated as well, using a small library for selected disk and spheroidal components by combining these spectra and re-scaling to give the magnitude sampled from the luminosity function.

\section{Firsts tests}

In order to verify how well JStuff is performing in relation to the original E. Bertin's C version, we simulated a small dataset, containing about 150,000 galaxies with the C version's default parameters. This first simulation indicates that our code is reproducing well most aspects of its predecessor (Fig. 1). The code is being modified to use a spectral library of galaxies, simulated with the PEGASE code at the University of Athens. As soon as this integration is finished, a strictier validation, using a larger simulated dataset will be performed. At this validation a comparison with real data is planned.

\section{References}

Altamirano, P., Babusiaux, C., Luri, X., \& Masana, E. 2005, Technical Report GAIA-SWG-004

Bertin, E. \& Arnouts, S. 1996, A\&AS, 117, 393

Binggeli, B., Sandage, A., \& Tarenghi, M. 1984, AJ, 89, 64

Erben, T. Van Waerbeke, L., Bertin, E., Mellier, Y., \& Schneider, P. 2001, A\&A, 366, 717

Metcalfe, N., Shanks, T., Fong, R., \& Jones, L. R. 1991, MNRAS, 249, 498

Perryman, M. 2005, The Three-Dimensional Universe with Gaia, ESA/SP-576

Sandage, A., Freeman, K. C., \& Stokes, N. R. 1970, ApJ, 160, 831

Schechter, P. 1976, ApJ, 203, 297

Simien, F. \& de Vaucouleurs, G. 1986, ApJ, 302, 564 